\title{
Density functional theory, chemical reactivity, and the Fukui functions
}

\author{
R. Pucci ${ }^{1,2}$ (D) G. G. N. Angilella ${ }^{1,2,3,4,5,6}$ (iD
}

Accepted: 10 October 2021 / Published online: 17 January 2022

(c) The Author(s) 2022

\begin{abstract}
We review the early works which were precursors of the Conceptual Density Functional Theory. Starting from Thomas-Fermi approximation and from the exact formulation of Density Functional Theory by Hohenberg and Kohn's theorem, we will introduce electronegativity and the theory of hard and soft acids and bases. We will also present a general introduction to the Fukui functions, and their relation with nucleophilicity and electrophilicity, with an emphasis towards the importance of these concepts for chemical reactivity.
\end{abstract}

Keywords Conceptual density functional theory $\cdot$ Chemical reactivity $\cdot$ Fukui functions

\section{Introduction}

Current interest in chemical reactivity is shared by both chemists, and physicists, biologists, as well as material scientists. This is due to the fact that chemical reactivity describes phenomena strongly connected with the response of a substance with respect to a specific chemical reaction. Existing empirical models have been therefore rationalized, and new ones have been introduced. In particular, models based on quantum mechanics, and more specifically on the so-called Conceptual Density Functional Theory (CDFT), are among the most credited and suggestive ones. Indeed, Density Functional Theory (DFT) allowed to determine regularities in atomic and molecular series (Pucci and March 1982, 1986a, b), which were otherwise impossible to detect within other, albeit accurate, computational

G. G. N. Angilella

giuseppe.angilella@ct.infn.it

R. Pucci

renato.pucci@ct.infn.it

1 Dipartimento di Fisica e Astronomia 'Ettore Majorana', Università di Catania, Via S. Sofia, 64, 95123 Catania, Italy

2 Accademia di Gioenia di Catania, Catania, Italy

3 CNR-IMM, Catania, Italy

4 INFN, Sez. Catania, Catania, Italy

5 CNISM, UdR Catania, Italy

6 Scuola Superiore di Catania, Università di Catania, Via Valdisavoia 9, 95123 Catania, Italy 
schemes, which could only be applied to individual cases. The local character of chemical reactivity has received more attention recently, as physical, as opposed to chemical, experimental tools have been devised to measure the coordination number in metal clusters on the atomic scale through atomic force microscopy (Berwanger et al. 2020).

In this paper, we will review the main physical concepts and theories underlying commonly used descriptors of chemical reactions, such as hardness and softness, especially with reference to acids and bases. We will start from Thomas-Fermi approximation, as a precursor of Density Functional Theory, which in turn allows a non controversial definition of elusive concepts such as the chemical potential and electronegativity. We will eventually emphasize the importance of the Fukui functions, and their role in determining the region of a molecule where it is more favourable to add or subtract an electron during a chemical reaction.

\section{Density functional theory}

Quantum calculations based on Schrödinger's equation have achieved a remarkable accuracy in the study of atoms and small molecules. However, in order to deal with molecules containing a relatively large number of electrons, as well as with solids, one can make recourse to an equally accurate theory, viz. Density Functional Theory (DFT). At the core of DFT stands the electron density $\rho(\mathbf{r})$, rather than the wave function $\Psi\left(\mathbf{r}_{1} \sigma_{1}, \mathbf{r}_{2} \sigma_{2}, \ldots \mathbf{r}_{N} \sigma_{N}\right)$, where $\mathbf{r}_{i}, \sigma_{i}$ are the space and spin coordinates, rescpectively, of the $i$-th electron in the system $(i=1, \ldots N)$. Single electron densities $\rho(\mathbf{r})$ can indeed be extracted experimentally in diffraction measurements, and is at the basis of a quantum theory of the system's ground state. The simplification brought about by a single-particle field, instead of a fully manybody wavefunction, is enormous (cf. March and Angilella 2016, for a more rigorous definition of $\rho$ in terms of $\Psi$, and for the more general concept of density matrix) .

A notable precursor to DFT was the Thomas-Fermi (TF) approximation (Thomas 1926; Fermi 1927), which we will now briefly summarize.

\section{Thomas-Fermi approximation}

Thomas (1926) and, independently, Fermi (1927) treated the electron cloud around an atomic nucleus as a fully degenerate electron gas (Pucci and Angilella 2006; March 1975; Pucci 1986). One can therefore establish a local relation between the electronic density $\rho(\mathbf{r})$ at position $\mathbf{r}$ with respect to the nucleus and the Fermi momentum $p_{\mathrm{F}}(\mathbf{r})$ :

$$
\rho(\mathbf{r})=2 \frac{4 \pi}{3} p_{\mathrm{F}}^{3}(\mathbf{r})
$$

where the factor of 2 takes into account for Pauli exclusion principle. The Fermi momentum $p_{\mathrm{F}}(\mathbf{r})$ at position $\mathbf{r}$ is related to the self-consistent potential $V(\mathbf{r})$ by the equation

$$
p_{\mathrm{F}}^{2}(\mathbf{r})=2 m\left[E_{\mathrm{F}}-V(\mathbf{r})\right],
$$

where $m$ is the electron mass and $E_{\mathrm{F}}$ is the Fermi energy. The latter can be obtained via the normalization condition 


$$
\int \rho(\mathbf{r}) d^{3} \mathbf{r}=N,
$$

where $N$ is the total number of electrons, equalling the atomic number $Z$ in a neutral atom. Inserting Eq. (2) into Eq. (1), making use of Poisson equation for electrostatics, and introducing the screening factor $\phi$, one obtains

$$
V(\mathbf{r})-E_{\mathrm{F}}=-\frac{Z e^{2}}{r} \phi(r) .
$$

Hence, one derives the dimensionless TF equation for a spherically symmetric electron distribution

$$
\frac{d^{2} \phi}{d x^{2}}=\frac{\phi^{3 / 2}}{x^{1 / 2}}
$$

where

$$
r=b x
$$

and $b$ is the scaling factor

$$
b=\frac{1}{4}\left(\frac{9 \pi^{2}}{2 Z}\right)^{1 / 3}=\frac{0.8853}{Z^{1 / 3}} a_{0},
$$

with $a_{0}$ the Bohr radius.

Equation (5) is 'universal', in the sense that the only dependence on the atomic number $Z$ is present in the scaling factor $b$. Within TF approximation, it suffices to solve Eq. (5) for a particular atom, while the solution for all other atoms can be obtained by simply scaling distance by $b$.

\section{Hohenberg and Kohn theorem}

An important generalization of the TF model was obtained by Dirac (1930) to include the exchange energy, again for a uniform electron gas. Some effort was also directed at including correlation effects (Gombás 1949; March 1957). Other attempts to improve the model were due to Majorana (Pucci and Angilella 2006). However, it was generally believed that the model was not founded on a sufficiently well established basis.

The attitude of the scientific community was to change dramatically in 1964, when Hohenberg and Kohn (1964) succeeded in providing Thomas-Fermi theory with a formal foundation. Let an arbitrary number $N$ of electrons be subject to an external potential $V_{\text {ext }}(\mathbf{r})$. Hohenberg and Kohn theorem then states that $V_{\text {ext }}(\mathbf{r})$ is a unique functional of the electron density $\rho(\mathbf{r})$, apart from an (irrelevant) additive constant. Since $V_{\text {ext }}(\mathbf{r})$ in turn specifies the Hamiltonian $H$ of the system, it follows that the ground-state energy of a many-electron system is a unique functional of $\rho(\mathbf{r})$.

Moreover, Hohenberg and Kohn theorem states that for all test densities $\bar{\rho}(\mathbf{r})$ such that $\bar{\rho}(\mathbf{r}) \geq 0$ and $\int \bar{\rho}(\mathbf{r}) d^{3} \mathbf{r}=N$, one has

$$
E_{0} \leq E_{v}[\bar{\rho}]
$$

where $E_{0}$ is the ground-state energy evaluated using the exact electronic density, and $E_{v}[\bar{\rho}]$ is the energy evaluated variationally using the test density $\bar{\rho}$. 


\section{The exact formulation of DFT}

Hohenberg and Kohn theorem allows to characterize in a formally exact way the total energy $E$ of the ground state through a variational principle with respect to the electronic density $\rho$ (Pucci 2017):

$$
\frac{\delta(E-\mu N)}{\delta \rho}=0,
$$

where $\mu$ is a Lagrange multiplier introduced to take into account for the normalization condition, Eq. (3). The exact expression for the ground-state energy $E \equiv E[\rho]$ is (March and Pucci 1984)

$$
E=T[\rho]+\int \rho V_{N} d^{3} \mathbf{r}+\frac{1}{2} \int \rho V_{e} d^{3} \mathbf{r}+V_{x c}[\rho],
$$

where $T$ is the total kinetic energy, including the kinetic correlation energy, as a functional of $\rho$, while $V_{x c}$ is the potential energy for exchange $(x)$ and correlation $(c)$ interactions. The second term in Eq. (10) is the potential energy for the electrons-nuclei interaction, while the third term is the interaction energy of the electron cloud with density $\rho(\mathbf{r})$. Formally, one may apply the variational principle Eq. (9) directly to Eq. (10), but, as has been emphasized by (Kohn and Sham 1965), it is often useful to introduce an equivalent singleelectron problem. This consists in dividing $T$ in two parts: a single-particle kinetic energy $T_{S}$, but corresponding to the exact electronic density $\rho(\mathbf{r})$, and a correlation kinetic energy which can be embedded within $V_{x c}$ in Eq. (10), in order to obtain a total exchange and correlation energy $E_{x c}\lfloor\rho$ ]. Thus the formally exact (single-electron) version of Euler equation becomes

$$
\begin{aligned}
\mu & =\frac{\delta T_{S}}{\delta \rho}+V_{\mathrm{H}}+\frac{\delta E_{x c}}{\delta \rho} \\
& =\frac{\delta T}{\delta \rho}+V_{\mathrm{H}}+\frac{\delta V_{x c}}{\delta \rho} .
\end{aligned}
$$

One may note, together with (Kohn and Sham 1965), that the first expression formally defines a one-body potential $V(\mathbf{r})$ given by

$$
V(\mathbf{r})=V_{\mathrm{H}}+\frac{\delta E_{x c}}{\delta \rho}
$$

where $V_{\mathrm{H}}(\mathbf{r})$ is Hartree potential, which may be obtained explicitly by direct comparison with Eq. (10).

Of course, an exact knowledge of the exchange and correlation potential $\delta E_{x c} / \delta \rho$ in Eq. (12) would require the exact solution of the full many-body problem in the inhomogeneous electron gas in an atom, molecule, or solid, which is at present beyond reach. Therefore, sensible progress in the solution to the problem can be achieved by 'reasonable' approximations to this term in Eq. (10). Presently, all calculations of the geometrical structure and of the band structure of solids are performed within DFT. Also, DFT is used to study various properties of several molecular systems. 
Fig. 1 Redrawn from Fig. 1 of March and Pucci (1983). Solid line is $|\mu|$, while dashed line is $|I+A| / 2$ for the isoelectronic sequence with $N=9$, both as a function of energy. March and Pucci (1983) make use of the Hartree-Fock results of (Clementi 1965) and of the $1 / Z$ development of Layzer (1959). All quantities are in atomic units

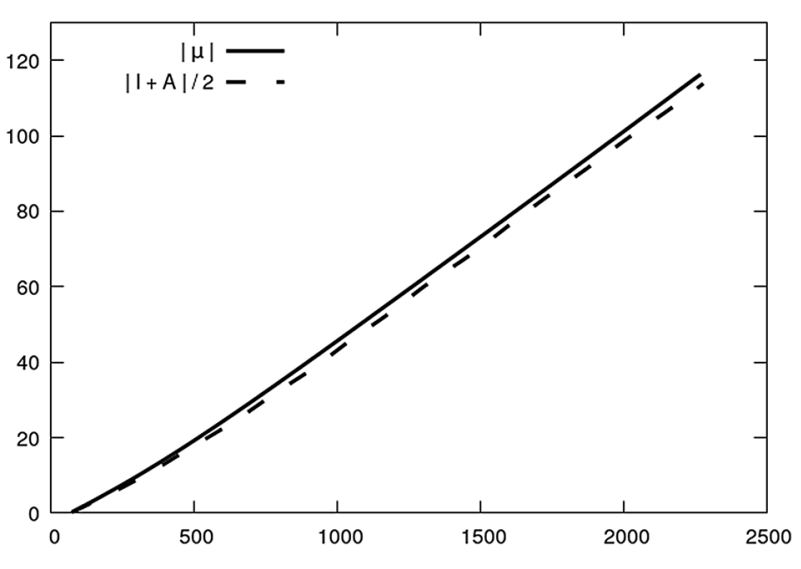

|E]

\section{Chemical potential and electronegativity}

The Lagrange multiplier introduced in Eq. (9) can only be accessed numerically with some difficulty, as it is a small quantity with respect to the total energy $E$. In addition, its physical meaning may strongly depend on the approximations. For instance, within the TF model, it coincides with the classical energy of the fastest electron. On the other hand, as of late, it has been possible to elucidate its physical meaning, taking into account of the effects of exchange and correlation. Such developments are important for the study of chemical reactivity.

Within statistical quantum theory of solids (where the number of electrons is as large as $N \approx 10^{23}$ ), energy can be thought as a continuous function of $N$, and $\mu$ coincides with the chemical potential:

$$
\mu=\frac{\partial E}{\partial N}
$$

A rigorous and size-consistent way to treat the number of the electrons $N$ as a continuous variable has been suggested by (Perdew et al. 1982) (see also Yang et al. 2000; Ayers 2008). On the other hand, this assumption breaks down for relatively smaller values of $N$, in which case $E$ cannot be treated as a continuous function of $N$. This gives rise to several fundamental difficulties in the study of systems with a finite $N$.

Several methods exist, however, which allow to derive some important conclusions on the physical meaning of $\mu$, by including effects of exchange and correlation. The first method is due to March and Pucci (1984), and derives from the fact that $\mu$ has the same numerical value at all points of the system under consideration. One can therefore evaluate $\mu$ also in the limit $r \rightarrow \infty$, to obtain

$$
\mu \approx-I+\lim _{r \rightarrow \infty} \frac{\delta E_{x c}}{\delta \rho},
$$

where $I$ is the ionization potential. One may argue that the second term in the right-hand side of Eq. (14) is related to the electronic affinity $A$. This fact is indeed confirmed by March and Pucci (1983), whose main result is redrawn in Fig. 1.

In March and Pucci (1984), it is also shown that, within Hartree-Fock theory, one has 
Fig. 2 Energy as a function of electron number for a specific atom or molecule $\mathrm{S}$. Slope is $d E / d N \approx(I+A) / 2$, while curvature is $d^{2} E / d N^{2} \approx I-A$. Redrawn after Parr and Yang (1989)

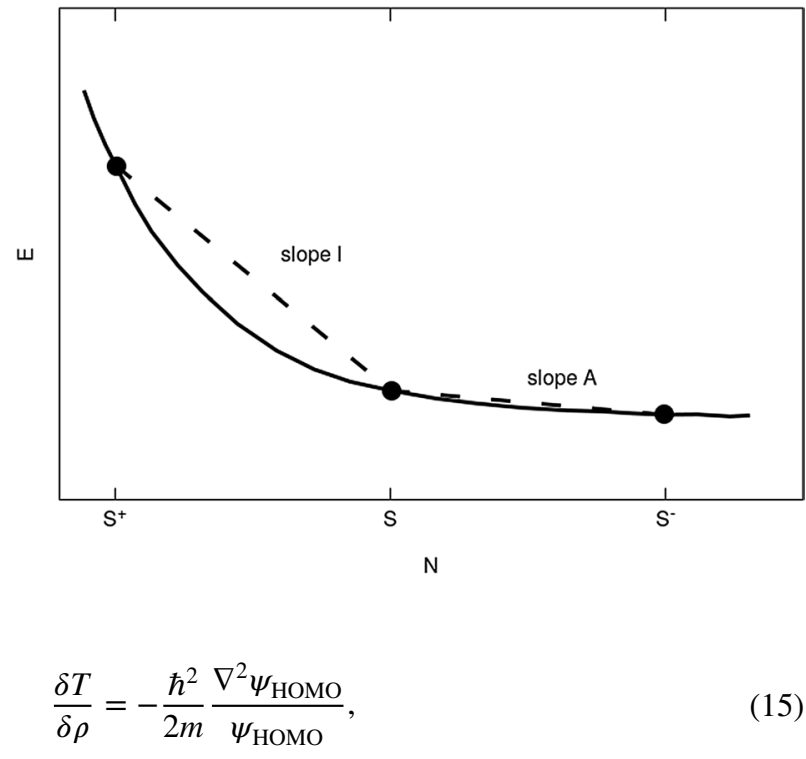

where $T$ is the total kinetic energy and $\psi_{\text {Hомо }}$ is the highest-occupied molecular orbital. Since the left-hand side of Eq. (15) is a functional of electronic density $\rho(\mathbf{r})$, while the right-hand side if an explicit functional of $\psi_{\mathrm{HOMO}}$, one concludes that $\rho=\rho\left[\psi_{\mathrm{HOMO}}\right]$. Moreover, one may prove that, again within Hartree-Fock theory, the density matrix $\rho\left(\mathbf{r}, \mathbf{r}^{\prime}\right)$ is determined only by $\psi_{\mathrm{HOMO}}(\mathbf{r})$. One may conclude therefore that within Hartree-Fock theory the ground-state energy $E_{\mathrm{HF}}$ is an, as yet undetermined, functional of the wavefunction $\psi_{\mathrm{HOMO}}(\mathbf{r})$. This latter consideration and Eq. (15) show that the frontier orbitals are of fundamental importance also within DFT.

The chemical potential of a many-electron assembly (atom or molecule of a given chemical species, in our context) is usually defined as

$$
\mu=\left(\frac{\partial E}{\partial N}\right)_{V} .
$$

A positive value of the chemical potential is therefore associated with the infinitesimal increase of energy when electronic charge is added to such a system. Several examples show that energy indeed decreases when electronic charge is added to a species (Fig. 2): consider e.g. the sequence $\mathrm{Li}^{+}, \mathrm{Li}, \mathrm{Li}^{-}$, for which QCISD(T)6-311+G* yield energies of $-7.23584,-7.43203$, and $-7.45448 h$, respectively (Lewars 2011).

The electronic chemical potential is thus the local slope of the energy curve as a function of the electron number (Chattaraj et al. 2002). Parr et al. (1978) employed finite differences to identify the chemical potential $\mu$ with the negative electronegativity:

$$
\mu=-\chi,
$$

where $\chi$ is Mulliken's electronegativity (Mulliken 1934),

$$
\chi=\frac{I+A}{2} .
$$

According to electronegativity, Eq. (18), atoms and molecules can be ranked on the basis of their ability to attract or lose electrons in the presence of atoms of another chemical 
species. Eq. (18) is therefore of fundamental importance in problems of chemical reactivity, even though historically it was introduced in an 'empirical' way (Mulliken 1934), and has been nowadays recovered within a well-founded theoretical framework.

The above definition of electronegativity was introduced by Iczkowski and Margrave (1961) and later discussed within the context of DFT by Parr et al. (1978). Looking at Fig. 2 and using finite-difference approximation to estimate slopes, one may define the ionization energy of a given chemical species $S$ as $I=E\left(S^{+}\right)-E(S)$, the electron affinity of $S$ as $A=E(S)-E\left(S^{-}\right)$, and summing up the previous two equations, $I+A=E\left(S^{+}\right)-E\left(S^{-}\right)$.

In the following, we will also discuss the equally important higher-order derivatives. We note preliminarly that both the chemical potential and the electronegativity satisfy the following conditions: (1) their value is the same everywhere in the system under consideration, and (2) when two systems are merged together, $A$ and $B$, say, with different electronegativities $\chi_{A}$ and $\chi_{B}$, respectively, (or different chemical potentials), the resulting system $C=A+B$ will be characterized by a single electronegativity $\chi_{C}$.

Electronegativity is one of the descriptors of chemical reactivity more employed to understand the nature of chemical interactions (Kaya et al. 2018). An important development in this direction has been achieved via Sanderson's electronegativity equalization principle (Sanderson 1971). This principle is based on the assumption that the properties of a molecule can be determined from those of the constituent atoms. Sanderson's principle states that, due to charge transfer, the electronegativity of a molecule $\chi_{M}$ comes from the equalization of the electronegativities of the constituent atoms:

$$
\chi_{M}=\left(\prod_{i=1}^{N} \chi_{i}\right)^{1 / N}
$$

where $\chi_{i}(i=1, \ldots N)$ stands for the electronegativity of the $i$ th isolated atom, and $N$ is the total number of atoms in the molecule. It is well known that such a 'group electronegativity' is an important tool for studying chemical reactivity. Later, we shall briefly refer to the importance of other equalization principles in the context of chemical reaction processes.

To conclude this section, let us mention the ' $|\Delta \mu|$ big is good' rule. Such a statement, referred to by Parr and Yang (1989), is related to the fact that, during a reaction between a reactant and two molecules having parts with similar disposition, the reactant prefers the molecule associated to the maximum answer of the chemical potential. Recently, MirandaQuintana et al. (2018b) have provided more support to this statement.

\section{Hardness and softness}

Following an initial anticipation by (Mulliken 1952), the chemical concepts of hardness and softness have been made widely known by Pearson's work (1963). In essence, the hardness or softness of a chemical species (atom, ion, or molecule) is a qualitative indication of its polarizability, i.e. of the tendency of its electron cloud to get distorted by an external electrical field. The terms 'hardness' and 'softness' probably originate from their use to indicate the resistance of a body towards deformation under a mechanical stress (cf. Lewars 2016, p. 497).

Although there has been no universal consensus on the precise meaning of 'hard' and 'soft' until recently (cf. Pearson 1966; Ayers 2005; Ayers et al. 2006), a species can be 
unequivocally characterized as being hard or soft. Following Yang and Parr (1985), it is possible to summarize these characteristics as follows:

1. Soft basis: The donor atom has a high polarizability and a low electronegativity, it can be oxidized easily and it is associated with empty low-energy orbitals.

2. Hard basis: The donor atom has a low polarizability and a high electronegativity, it cannot be easily oxidized and it is associated with empty high-energy orbitals.

3. Soft acids: The acceptor atom has a low electric charge, relatively large dimensions, and its external electrons can be easily excited.

4. Hard acids: The acceptor atom has a high electric charge, relatively small dimensions, and its external electrons cannot be easily excited.

From his phenomenological description, Pearson (1963) also derived the principle according to which hard acids prefer to get bonded with hard bases, and soft acids prefer to get bonded with soft bases.

Twenty years later, Parr and Pearson (1983) identified hardness $\eta$ with the second-order derivative of electronic energy $E$ with respect to the electron number $N$ :

$$
\eta=\frac{1}{2} \frac{\partial^{2} E}{\partial N^{2}}=\frac{1}{2} \frac{\partial \mu}{\partial N}
$$

where derivatives are taken at constant external potential. Pearson noted that 'There seems to be a rule of nature that molecules arrange themselves so as to be as hard as possible' (Pearson 1987). This statement led to the establishment of the maximum hardness principle. The above concepts lie at the heart of what is nowadays called Conceptual Density Functional Theory (CDFT) (Miranda-Quintana et al. 2018a). Going to finite differences (cf. Fig. 2), one finds that

$$
\eta=\frac{I-A}{2}
$$

The inverse of hardness is softness $S$ (Yang and Parr 1985),

$$
S=\frac{1}{\eta}=\frac{\partial N}{\partial \mu} .
$$

The definitions of hardness and softness given in Eqs. (21) and (22), respectively, are said 'global', since they are related to the molecule on the overall. However, it is also possible to introduce a local concept of hardness and softness, as we will see in the following.

Let us note that $\mu$ and $\eta$ can be identified from the second-order Taylor expansion for the energy variation $\Delta E$ of an electron system from one fundamental state to another:

$$
\Delta E=\left(\frac{\partial E}{\partial N}\right)_{V(\mathbf{r})} \Delta N+\frac{1}{2} \frac{\partial^{2} E}{\partial N^{2}}(\Delta N)^{2} .
$$

For instance, this formula applies to the control of charge transfer where the system geometry stays approximately constant (Miranda-Quintana 2018). In any event, from the identification of the most favorable changes (i.e. those inducing a more negative, or less positive, value of $\Delta E$ ), one may obtain information on the chemical reaction under consideration.

To conclude this section, we note that in addition to those already mentioned, there are other descriptors of reactivity, one example being the global electrophilicity index $\omega$. This 
can be defined as the stabilization energy gained by a system when it reaches equilibrium, namely

$$
\omega=\frac{\mu^{2}}{2 \eta}
$$

In this case, the system acquires from the surrounding environment an additional electronic charge $\Delta N$ given by (Sablon et al. 2007)

$$
\Delta N_{\max }=-\frac{\mu}{\eta} .
$$

\section{Fukui functions}

In 1984, Yang, Parr, and Pucci first introduced the term 'Fukui functions' 1 in a paper entitled Electron density, Kohn-Sham frontier orbitals and Fukui functions (Yang et al. 1984a) (cf. also Yang et al. 1984b). Actually, several earlier works made use of frontier orbitals in connection with DFT. In particular, within the present context, we would like to remind the paper by Parr and Yang (1984), which was submitted a few months before Yang et al. (1984a). Yang et al. (1984a) define the Fukui function as the variation in the electronic density due to the change in the electron number at fixed external potential:

$$
f(\mathbf{r})=\left(\frac{\partial \rho(\mathbf{r})}{\partial N}\right)_{V(\mathbf{r})} .
$$

Chemical reactions imply some charge transfer among the various parts of the reacting molecules. Local properties are thus essential in the description of the reactivity of molecular systems. It is necessary to identify which is the most favorable location to remove or add an electron to a molecule. Therefore it is extremely important to determine the variation of the electronic density under the influence of an approaching reactant. To this purpose, let us remind that by nucleophilicity one means the ability of a reactant to make its electrons available with an electrophile species in the course of a reaction. In other words, reactants looking for electrons are termed electrophile, while reactants that donate electrons are termed nucleophile.

In Eq. (26), one has to note that $f(\mathbf{r})$ has different values if the derivative is taken from either direction. It has been demonstrated (Yang et al. 1984a) that, within Janak's (1978) generalization of Kohn-Sham's theory, one has

$$
\begin{aligned}
& f^{+}(\mathbf{r})=\left|\phi_{\mathrm{LUMO}}(\mathbf{r})\right|^{2}+\sum_{i \geq \mathrm{LUMO}}\left(\frac{\partial\left|\phi_{i}(\mathbf{r})\right|^{2}}{\partial N}\right)_{V(\mathbf{r})}, \\
& f^{-}(\mathbf{r})=\left|\phi_{\mathrm{HOMO}}(\mathbf{r})\right|^{2}+\sum_{i \leq \mathrm{HOMO}}\left(\frac{\partial\left|\phi_{i}(\mathbf{r})\right|^{2}}{\partial N}\right)_{V(\mathbf{r})},
\end{aligned}
$$

\footnotetext{
1 Kenichi Fukui was born in Nora, Japan, in 1918. He was professor at the Imperial University of Kyoto from 1951, received the Nobel Prize in 1981, passed away in 1998.
} 
Fig. 3 Contour lines of the Fukui function $f^{+}(\mathbf{r})$ in $\mathrm{H}_{2} \mathrm{CO}$. Along the vertical axis, $z$ denotes the coordinate perpendicular to the molecular plane. The carbon atom is located at -1.015 a.u., while the oxygen atom is located at 1.27 a.u. Schematically redrawn after Parr and Yang (1989), p. 100

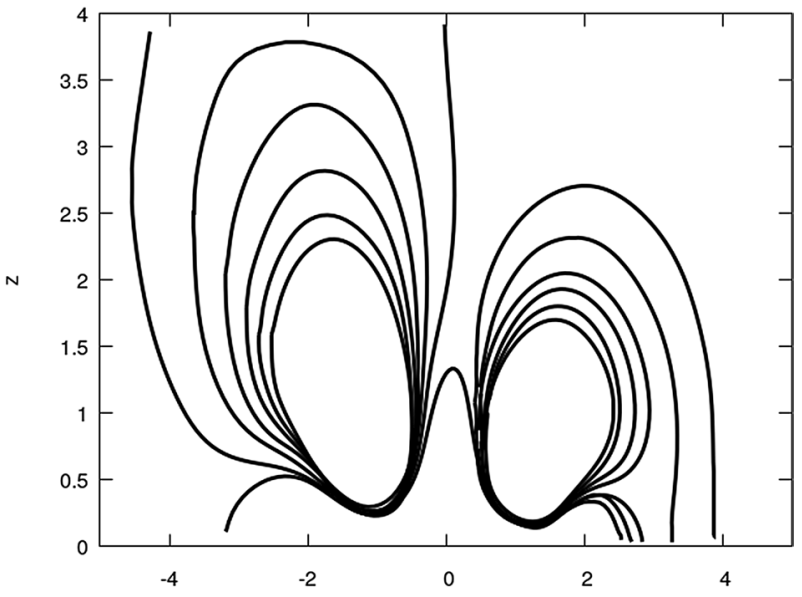

where the index $i$ runs over all the orbitals in Kohn-Sham's equations, LUMO stands for lowest-unoccupied molecular orbital, and HOMO for highest-occupied molecular orbital. The second term in the right-hand sides of Eqs. (27) is not included in the theory of the frontier orbitals by (Fukui 1975), and takes into accout for orbital relaxation and electronic correlation. These effects are important, for instance, in reactions which imply electron transfer induced by oxidoreduction (Echegaray et al. 2017).

Moreover it has been noted that within the canonical ensemble at zero temperature the Fukui function for a nucleophile attack is given by (Echegaray et al. 2017):

$$
f^{+}(\mathbf{r})=\rho_{N+1}(\mathbf{r})-\rho_{N}(\mathbf{r}),
$$

if the derivative is taken from the right, and by

$$
f^{-}(\mathbf{r})=\rho_{N}(\mathbf{r})-\rho_{N-1}(\mathbf{r}),
$$

if the derivative is taken from the left, and is related to an electrophile attack. For a neutral (i.e. radical) attack, one has instead:

$$
f^{0}(\mathbf{r})=\frac{1}{2}\left(\rho_{N_{0}+1}(\mathbf{r})+\rho_{N_{0}-1}(\mathbf{r})\right) .
$$

The Fukui functions have a local character, and therefore contour lines can be drawn for them in specific cases, as proposed by Lee et al. (1988) and shown, for instance, in Fig. 3 for the $\mathrm{H}_{2} \mathrm{CO}$ molecule (see also Ayers et al. 2007; Geerlings and De Proft 2008; Komorowski et al. 2011). These maps allow to identify which sites in a molecule are more favorable for a nucleophile, or an electrophile attack.

\section{Local hardness and softness}

Yang and Parr (1985) surmised that the concepts of hardness and softness are intimately related to the Fukui functions. Therefore they provided local defitions thereof. Actually, the Fukui function $f(\mathbf{r})$ and the local softness $s(\mathbf{r})$ are very often used as local reactivity parameters. Yang and Parr (1985) then define local softness as 


$$
s(\mathbf{r})=\left(\frac{\partial \rho(\mathbf{r})}{\partial N}\right)_{V}\left(\frac{\partial N}{\partial \mu}\right)_{V}=f(\mathbf{r}) S,
$$

where $S$ is the global softness defined in Eq. (22). The local softness is the inverse of the local hardness (Berkowitz and Parr 1988) and enables to locate the specific reaction sites between two reactants.

\section{Conclusions}

We have reviewed some of the works which led towards the development of Conceptual Density Functional Theory in problems of chemical reactivity. Recent developments of these studies (Kaya et al. 2018; Miranda-Quintana et al. 2018b, a; Echegaray et al. 2017; Lamine et al. 2019; Fuentealba et al. 2016; Gázquez et al. 2018; Coulibaly et al. 2019; Lee et al. 2019; Sánchez-Márquez et al. 2018) demonstrate the ongoing interest in this field. Novel and more realistic models will allow to predict reactivity indices with increasing accuracy.

Acknowledgements RP wishes to thank the late Norman H. March and Bob Parr, for numerous and deep discussions over the general field embraced by the present review. GGNA also acknowledges partial support from the University of Catania, through 'Piano di Incentivi per la Ricerca di Ateneo 2020/2022', proposal Q-ICT.

Open Access This article is licensed under a Creative Commons Attribution 4.0 International License, which permits use, sharing, adaptation, distribution and reproduction in any medium or format, as long as you give appropriate credit to the original author(s) and the source, provide a link to the Creative Commons licence, and indicate if changes were made. The images or other third party material in this article are included in the article's Creative Commons licence, unless indicated otherwise in a credit line to the material. If material is not included in the article's Creative Commons licence and your intended use is not permitted by statutory regulation or exceeds the permitted use, you will need to obtain permission directly from the copyright holder. To view a copy of this licence, visit http://creativecommons.org/licenses/by/4.0/.

\section{References}

Angilella, G.G.N., La Magna, A. (eds.): Correlations in Condensed Matter Under Extreme Conditions: A Tribute to Renato Pucci on the Occasion of His 70th Birthday. Springer Verlag, New York (2017). Ebook: 9783319536644

Ayers, P.W.: An elementary derivation of the hard/soft-acid/base principle. J. Chem. Phys. 122, 141102 (2005)

Ayers, P.W.: The dependence on and continuity of the energy and other molecular properties with respect to the number of electrons. J. Math. Chem. 43, 285-303 (2008)

Ayers, P.W., De Proft, F., Borgoo, A., Geerlings, P.: Computing Fukui functions without differentiating with respect to electron number. I. Fundamentals J. Chem. Phys. 126, 224107 (2007)

Ayers, P.W., Parr, R.G., Pearson, R.G.: Elucidating the hard/soft acid/base principle: a perspective based on half-reactions. J. Chem. Phys. 124, 194107 (2006)

Berkowitz, M., Parr, R.G.: Molecular hardness and softness, local hardness and softness, hardness and softness kernels, and relations among these quantities. J. Chem. Phys. 88, 2554-2557 (1988)

Berwanger, J., Polesya, S., Mankovsky, S., Ebert, H., Giessibl, F.J.: Atomically resolved chemical reactivity of small Fe clusters. Phys. Rev. Lett. 124, 096001 (2020)

Chattaraj, P.K., Paddar, A., Maiti, B.: Chemical reactivity and dynamics within a density-based quantum mechanical framework. In: Sen, K.D. (ed.) A Celebration of the Contributions of Robert G Parr, volume 2 of Reviews of Modern Chemistry. World Scientific, Singapore (2002)

Clementi, E.: Tables of atomic functions. IBM J. Res. Develop. Suppl. 9, 2 (1965) 
Coulibaly, W.K., N‘dri, J.S., Konè, M.G.-R., Dago, C.D., N‘ta Ambeu, C., Bazureau, J.-P., Ziao, N.: Studies of the chemical reactivity of a series of rhodanine derivatives by approaches to quantum chemistry. Comp. Mol. Biosci. 9, 49 (2019)

Dirac, P.A.M.: Note on exchange phenomena in the Thomas atom. Proc. Cambridge Phil. Soc. 26, 376-385 (1930)

Echegaray, E., Toro-Labbe, A., Dikmenli, K., Heidar-Zadeh, F., Rabi, N., Rabi, S., Ayers, P.W., Cárdenas, C., Parr, R.G., Anderson, J. S.M.: Negative condensed-to-atom Fukui functions: a signature of oxidation-induced reduction of functional groups. In: Angilella and La Magna (2017), chapter 19, pp. 269-288. Ebook: 9783319536644

Fermi, E.: Un metodo statistico per la determinazione di alcune proprietà dell'atomo. Rendiconti dell'Accademia Nazionale dei Lincei 6, 602 (1927)

Fuentealba, P., Cardenas, C., Pino-Rios, R., Tiznado, W.: Topological analysis of the Fukui function. In: Chauvin, R., Lepetit, C., Silvi, B., Alikhani, E. (eds.) Applications of Topological Methods in Molecular Chemistry, volume 22 of Challenges and Advances in Computational Chemistry and Physics, chapter 8, pp. 227-241. Springer, Cham (2016)

Fukui, K.: Theory of Orientation and Stereoselection. Springer, Berlin (1975)

Gázquez, J.L., Franco-Pérez, M., Ayers, P.W., Vela, A.: Temperature-dependent approach to chemical reactivity concepts in density functional theory. Int. J. Quantum Chem. 119, e25797 (2018)

Geerlings, P., De Proft, F.: Conceptual DFT: the chemical relevance of higher response functions. Phys. Chem. Chem. Phys. 10, 3028-3042 (2008)

Gombás, P.: Die statistische Theorie des Atoms und ihre Anwendungen. Springer-Verlag, Vienna (1949)

Hohenberg, P.C., Kohn, W.: Inhomogeneous electron gas. Phys. Rev. 136, B864-B871 (1964)

Iczkowski, R.P., Margrave, J.L.: Electronegativity. J. Am. Chem. Soc. 83, 3547-3551 (1961)

Janak, J.F.: Proof that $\partial E / \partial n_{i}=\epsilon$ in density-functional theory. Phys. Rev. B 18, 7165-7168 (1978)

Kaya, S., Kaya, C., Obot, I.B.: Chemical equalization principles and their new applications. In: Islam, N., Kaya, S. (eds.) Conceptual Density Functional Theory and Its Application in the Chemical Domain, chapter 5. Apple Academic Press, New York (2018)

Kohn, W., Sham, L.J.: Self-consistent equations including exchange and correlation effects. Phys. Rev. 140, A1133-A1138 (1965)

Komorowski, L., Lipiński, J., Szarek, P., Ordon, P.: Polarization justified Fukui functions: the theory and applications for molecules. J. Chem. Phys. 135, 014109 (2011)

Lamine, W., Boughdiri, S., Christ, L., Merzoud, L., Morell, C., Chermette, H.: Relaxation of Kohn-Sham orbitals of organometallic complexes during the approach of a nucleophilic reactant (or an electron approach): the case of [sal(ph)en $]_{2} \mathrm{Zn}$ complexes. Theor. Chem. Accounts 139, 7 (2019)

Layzer, D.: On a screening theory of atomic spectra. Ann. Phys. 8, 271-296 (1959)

Lee, C., Yang, W., Parr, R.G.: Local softness and chemical reactivity in the molecules $\mathrm{CO}, \mathrm{SCN}^{-}$and $\mathrm{H}_{2}$ CO. J. Mol. Struct. Theochem. A Collection of Invited Papers Dedicated to Michael J.S. Dewar on the Occasion of His 70th Birthday 163, 305-313 (1988)

Lee, J., Bertels, L.W., Small, D.W., Head-Gordon, M.: Kohn-Sham density functional theory with complex, spin-restricted orbitals: accessing a new class of densities without the symmetry dilemma. Phys. Rev. Lett. 123, 113001 (2019)

Lewars, E.G.: Computational Chemistry: Introduction to the Theory and Applications of Molecular and Quantum Mechanics. Springer, New York (2011)

Lewars, E.G.: Computational Chemistry. Springer, New York (2016)

March, N.H.: The Thomas-Fermi approximation in quantum mechanics. Adv. Phys. 6, 1-101 (1957)

March, N.H.: Self-Consistent Fields in Atoms. Pergamon Press, Oxford (1975)

March, N.H., Angilella, G.G.N.: Exactly Solvable Models in Many-Body Theory. World Scientific, Singapore (2016)

March, N.H., Pucci, R.: Chemical potential related to total energy in isoelectronic sequences of positive ions. J. Chem. Phys. 78, 2480-2484 (1983)

March, N.H., Pucci, R.: Density, total energy and chemical potential of atomic ions and some molecules. In: Avery, J., Dahl, J.P. (eds.) Local Density Approximations in Quantum Chemistry and Solid State Physics, pp. 53-74. Plenum Press, New York (1984)

Miranda-Quintana, R.A.: Computing the unconstrained local hardness. In: Islam, N., Kaya, S. (eds.) Conceptual Density Functional Theory and Its Application in the Chemical Domain, chap. 2, p. 15. Apple Academic Press, New York (2018)

Miranda-Quintana, R.A., Franco-Pérez, M., Gázquez, J.L., Ayers, P.W., Vela, A.: Chemical hardness: temperature dependent definitions and reactivity principles. J. Chem. Phys. 149, 124110 (2018)

Miranda-Quintana, R.A., Heidar-Zadeh, F., Ayers, P.W.: Elementary derivation of the ' $|\Delta \mu|$ Big Is Good" rule. J. Phys. Chem. Lett. 9, 4344-4348 (2018) 
Mulliken, R.S.: A new electroaffinity scale; together with data on valence states and on valence ionization potentials and electron affinities. J. Chem. Phys. 2, 782-793 (1934)

Mulliken, R.S.: Molecular compounds and their spectra. ii. J. Am. Chem. Soc. 74, 811-824 (1952)

Parr, R.G., Donnelly, R.A., Levy, M., Palke, W.E.: Electronegativity: the density functional viewpoint. J. Chem. Phys. 68, 3801-3807 (1978)

Parr, R.G., Pearson, R.G.: Absolute hardness: companion parameter to absolute electronegativity. J. Am. Chem. Soc. 105, 7512-7516 (1983)

Parr, R.G., Yang, W.: Density functional approach to the frontier-electron theory of chemical reactivity. J. Am. Chem. Soc. 106, 4049-4050 (1984)

Parr, R.G., Yang, W.: Density Functional Theory of Atoms and Molecules. Oxford University Press, Oxford (1989)

Pearson, R.G.: Hard and soft acids and bases. J. Am. Chem. Soc. 85, 3533-3539 (1963)

Pearson, R.G.: Acids and bases. Science 151, 172-177 (1966)

Pearson, R.G.: Recent advances in the concept of hard and soft acids and bases. J. Chem. Ed. 64, 561 (1987)

Perdew, J.P., Parr, R.G., Levy, M., Balduz, J.L.: Density-functional theory for fractional particle number: derivative discontinuities of the energy. Phys. Rev. Lett. 49, 1691-1694 (1982)

Pucci, R.: Nuove metodologie comuni tra fisica e chimica teorica: la teoria del funzionale della densità. Giornale di Fisica 27, 256 (1986)

Pucci, R.: Novel common methodologies between physics and theoretical chemistry: Density functional theory. In: ngilella and La Magna (2017), chapter 21, pp. 289-301. Ebook: 9783319536644

Pucci, R., Angilella, G. G. N. Majorana: from atomic and molecular, to nuclear physics. Found. Phys. 36, 1554-1572 (2006). Preprint arXiv:physics/0605226

Pucci, R., March, N.H.: Total energy of atomic ions related to low-order ionization potentials and to diamagnetic susceptibility. J. Chem. Phys. 76, 6091-6094 (1982)

Pucci, R., March, N.H.: Equilibrium-bond-length predictions of very heavy heteronuclear molecules. Phys. Rev. A 33, 3511-3514 (1986)

Pucci, R., March, N.H.: Generalized 1/Z expansion for heteronuclear molecules. Int. J. Quantum Chem. 29, 949-958 (1986)

Sablon, N., De Proft, F., Ayers, P. W., Geerlings, P.: Computing Fukui functions without differentiating with respect to electron number. II. Calculation of condensed molecular Fukui functions. J. Chem. Phys. 126, 224108 (2007)

Sánchez-Márquez, J., García, V., Zorrilla, D., Fernández, M.: New insights in conceptual DFT: new model for the calculation of local reactivity indices based on the Sanderson's principle. Int. J. Quantum Chem. 119, e25844 (2018)

Sanderson, R.T.: Chemical bonds and bond energy. Academic Press, New York (1971)

Thomas, L.H.: The calculation of atomic fields. Math. Proc. Cambridge Phil. Soc. 23, 542-548 (1926)

Yang, W., Parr, R.G.: Hardness, softness, and the Fukui function in the electronic theory of metals and catalysis. Proc. Natl. Acad. Sci. 82, 6723-6726 (1985)

Yang, W., Parr, R.G., Pucci, R.: Electron density, Kohn-Sham frontier orbitals, and Fukui functions. J. Chem. Phys. 81, 2862-2863 (1984)

Yang, W., Parr, R.G., Pucci, R.: Electron density, Kohn-Sham frontier orbitals, and Fukui functions. J. Chem. Phys. 81, 2862-2863 (1984b). Reprinted as chap. 22, p. 303, of Ref. Angilella and La Magna (2017)

Yang, W., Zhang, Y., Ayers, P.W.: Degenerate ground states and a fractional number of electrons in density and reduced density matrix functional theory. Phys. Rev. Lett. 84, 5172-5175 (2000)

Publisher's Note Springer Nature remains neutral with regard to jurisdictional claims in published maps and institutional affiliations. 